\title{
Right ideal Howson semigroups
}

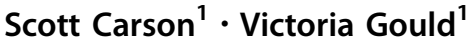

Received: 25 March 2020 / Accepted: 21 May 2020/Published online: 7 August 2020

(C) The Author(s) 2020

\begin{abstract}
We define a semigroup $S$ to be right ideal Howson if the intersection of any two finitely generated right ideals, or, equivalently, any two principal right ideals, is again finitely generated. We give many examples of such semigroups, including right coherent monoids, finitely aligned semigroups, and inverse semigroups. We investigate the closure of the class of right ideal Howson semigroups under algebraic constructions. For any $n \in \mathbb{N}^{0}$ we give a presentation of a right ideal Howson semigroup possessing an intersection of principal right ideals that requires exactly $n$ generators that is, in a particular sense, universal. We give analogous presentations for commutative, and for commutative cancellative, (right) ideal Howson semigroups.
\end{abstract}

Keywords Semigroup · Monoid · One-sided ideal · Presentations

\section{Introduction}

An algebra exhibits the Howson property if the intersection of two finitely generated subalgebras is also finitely generated. This term is in honour of the author of [15], who showed that the intersection of finitely generated subgroups of free groups is finitely generated. There have been a number of investigations of the Howson property for other classes of algebras. In particular, the Howson property for inverse semigroups has been studied by several authors such as Jones and Trotter $[16,17]$, Lawson and Vdovina [20] and Silva and Soares [25]. By contrast to the situation for

Communicated by Mark V. Lawson.

The first author acknowledges the support of EPSRC in the form of a Ph.D. studentship.

Victoria Gould

victoria.gould@york.ac.uk

Scott Carson

sc1485@york.ac.uk

1 Department of Mathematics, University of York, York YO10 5DD, UK 
groups, free inverse semigroups have the Howson property if and only if they are free on a one-element set [17].

The aim of this article is to change tack and to consider the Howson property for semigroups regarded as semigroup acts over themselves, so that the right (left) subacts of a semigroup $S$ are precisely its right (left) ideals. We consider $\emptyset$ as being a right (left) ideal with empty set of generators.

Definition 1.1 A semigroup $S$ is right (left) ideal Howson if the intersection of any two finitely generated right (left) ideals of $S$ is finitely generated.

Note that since intersection distributes over union, a semigroup is right (left) ideal Howson if and only if the intersection of principal right (left) ideals is finitely generated; we use this fact throughout this article. We remark that a monoid is right ideal Howson if and only if it is finitely aligned [7]; for semigroups being finitely aligned is a stronger condition, as we demonstrate. From this point we will explicitly refer to and give results for right ideal Howson semigroups; clearly, the dual results hold for left ideal Howson semigroups. Certainly for a commutative semigroup, the notions of right ideal Howson and left ideal Howson coincide; similar remarks apply to related definitions.

The property of being right ideal Howson is a finiteness condition for a semigroup; that is to say any finite semigroup is right ideal Howson. In Sect. 3 we show how it is connected to other finiteness conditions that have been studied recently, such as that of being right coherent [10, 11] or right Noetherian [22].

Semigroups that are right ideal Howson abound. We list some examples here, that may easily be verified by consulting any standard semigroup text such as [5, 14]: groups, inverse semigroups, completely (0-)simple semigroups, free semigroups and free monoids. We present many others subsequently in this paper. The reader may note that any of the semigroups in the previous list display the extra condition that the intersection of principal right ideals is empty or principal. Monoids that satisfy this extra condition have been well-studied by Clifford, Cherubini and Petrich: the latter authors referring to this condition (for left ideals) as Clifford's condition [3]. Indeed, Clifford [4] showed that bisimple inverse monoids can be viewed as inverse hulls of right cancellative monoids satisfying Clifford's condition. This connection has been developed by a number of authors such as Lawson [19], McAlister [21] and Reilly [24]. The notion of being finitely aligned [7] is closely connected with that of being right ideal Howson, being a stronger condition (Lemma 2.1); and coincides for many semigroups, including monoids. Indeed, it is noted in [7] that finitely aligned semigroups may be called right Howson. However, the situation for semigroups is more subtle; as we show in Remark 5.8, a right ideal Howson semigroup need not be finitely aligned. We introduce the term right ideal Howson to distinguish from that of being finitely aligned, and to make clear we are talking of right ideals and not right congruences. Explicit connections between finitely aligned semigroups, higher rank graphs and constructions of $C^{*}$-algebras are given in [7].

This article has several aims. One is to give natural, universal, examples of right ideal Howson semigroups such that the intersection of principal right ideals may 
require any fixed, finite number of generators. The second is to examine closure properties of the class of right ideal Howson semigroups under standard algebraic and semigroup theoretic operations. The third is to consider connections with other natural finiteness conditions. In this way we build a broader understanding of the class of right ideal Howson semigroups.

We organise this paper as follows. In Sect. 2 we recall essential terminology and fundamental results. In Sect. 3 we provide examples of right ideal Howson semigroups, with a particular focus on bands and coherent monoids. For each variety of bands, we give an explicit presentation of a right ideal Howson band belonging to the variety, and show that the lattice of varieties of bands splits into two with regard to Clifford's condition. We show that any semigroup given by a commutative presentation with finite set of relations is (right) coherent, and hence certainly (right) ideal Howson. In Sect. 4 we explore a number of closure results for the classes of right ideal Howson monoids and semigroups. We show that both the classes of right ideal Howson semigroups and right ideal Howson monoids are closed under free products. Right ideal Howson semigroups are not closed under direct products but, on the other hand, right ideal Howson monoids are closed under direct but not semidirect products. Finally, in Sect. 5, we consider a number of semigroup presentations, reflecting those given for bands in Sect. 3. We give presentations of right ideal Howson semigroups (which are also cancellative), commutative (right) ideal Howson semigroups and commutative cancellative (right) ideal Howson semigroups, all of which are universal in a given sense.

\section{Preliminaries}

We denote the natural numbers by $\mathbb{N}$ and put $\mathbb{N}^{0}=\mathbb{N} \cup\{0\}$. For any $n \in \mathbb{N}$ we define $\underline{n}:=\{1, \ldots, n\}$ and $\underline{n}^{0}:=\underline{n} \cup\{0\}$. Throughout this paper $S$ denotes a semigroup and $S^{1}$ is the monoid obtained from $S$ by adjoining an identity if necessary (so that $S^{1}=S$ if and only if $S$ is a monoid). For any element $a \in S$, the principal right ideal generated by $a$ is $a S^{1}=\{a\} \cup a S$, so that $a S^{1}=a S$ if and only if $a \in a S$. A right ideal is finitely generated if it is the finite union of principal right ideals. Our first observation will be useful in what follows.

Lemma 2.1 Let $S$ be a semigroup such that for all $a, b \in S$ we have

$$
a S \cap b S=u_{1} S \cup \cdots \cup u_{n} S
$$

where $u_{i} \in a S^{1} \cap b S^{1}$ for all $i \in \underline{n}$. Then $S$ is right ideal Howson.

Proof Let $S$ be as given. Then, for any $a, b \in S$, we may write

$$
a S^{1} \cap b S^{1}=(\{a\} \cap\{b\}) \cup(\{a\} \cap b S) \cup(a S \cap\{b\}) \cup(a S \cap b S) .
$$

Clearly, if $a \leq_{\mathscr{R}} b$ then $a S^{1} \cap b S^{1}=a S^{1}$ (the case where $b \leq_{\mathscr{R}} a$ is entirely dual). However, if $a \Varangle_{\mathscr{R}} b$ and $b \Varangle_{\mathscr{R}} a$ then we have by the above that $a S^{1} \cap b S^{1}=a S \cap b S$. Therefore, for such an $a, b \in S$, we have 


$$
a S^{1} \cap b S^{1}=u_{1} S \cup \cdots \cup u_{n} S \subseteq u_{1} S^{1} \cup \cdots \cup u_{n} S^{1} \subseteq a S^{1} \cap b S^{1}
$$

which gives us that $a S^{1} \cap b S^{1}=u_{1} S^{1} \cup \cdots \cup u_{n} S^{1}$ as required.

Semigroups satisfying the hypothesis of Lemma 2.1 are called finitely aligned in [7]. However, as we show in Remark 5.8, a right ideal Howson semigroup need not be finitely aligned. For semigroups that are right factorisable, that is, semigroups $S$ such that $s S=s S^{1}$ for any $s \in S$, the two notions coincide. This is the situation for monoids, inverse semigroups and bands (semigroups of idempotents), for example, but not for free semigroups.

The next observation we make follows quickly from the definition of a semigroup being right ideal Howson.

Lemma 2.2 A semigroup $S$ is right ideal Howson if and only if the monoid $S^{1}$ is right ideal Howson.

We will say a right ideal $I$ of $S$ is exactly $n$-generated for some $n \in \mathbb{N}^{0}$, if there are $n$ elements of $S$ that generate $I$, but no $n-1$ elements will suffice. With this in mind, we note that $I=\emptyset$ if and only if $I$ is exactly 0 -generated.

Definition 2.3 A semigroup $S$ satisfies (Rn) for $n \in \mathbb{N}^{0}$ if there exists some $a, b \in S$ such that $a S^{1} \cap b S^{1}$ is exactly $n$-generated. The condition ( $\left.L n\right)$ is defined dually.

\subsection{Semigroup presentations}

For an equivalence relation $\theta$ on a set $A$ we denote the $\theta$-class of $a \in A$ by $[a]_{\theta}$, or $[a]$ if $\theta$ is understood.

Let $X$ be a non-empty set, whose elements we will refer to as letters. We denote by $X^{+}\left(X^{*}\right)$ the free semigroup (free monoid) on $X$. We take $X^{+}$to be the set of all non-empty words over $X$ with operation concatenation, and to obtain $X^{*}$ we adjoin the empty word, often denoted by $\epsilon$.

The length of a word $w \in X^{*}$, denoted by $|w|$, is the number of letters in $w$, counting repeats. The context of $w$, written $c(w)$, is the set of letters that appear in $w$. For a number of results in this paper, it will be useful to let $\alpha(w)$ and $\omega(w)$ denote respectively the first and last letter of $w \in X^{+}$.

For a subset $\rho$ of $X^{+} \times X^{+}$, the smallest congruence relation on $X^{+}$containing $\rho$ is denoted by $\rho^{\sharp}$. For any $w, x \in X^{+}$we have that $(w, x) \in \rho^{\sharp}$ if and only if $w=x$ or there exists a finite sequence of the form

$$
w=z_{0}, z_{1}, \ldots, z_{n}=x
$$

where $z_{i-1}=c_{i} p_{i} d_{i}$ and $z_{i}=c_{i} q_{i} d_{i}$ with $\left(p_{i}, q_{i}\right) \in \rho \cup \rho^{-1}$ and $c_{i}, d_{i} \in X^{*}$ for all $i \in \underline{n}$ [14]. We say that $z_{i-1} \rightarrow z_{i}$ an elementary $\rho$-transition. The $\rho^{\sharp}$-class $[w]_{\rho^{\sharp}}$ of $w \in X^{+}$will be denoted by $[w]_{\rho}$ (or $[w]$ if $\rho$ is understood).

Definition 2.4 A semigroup presentation $\langle X: \rho\rangle$ consists of non-empty set $X$ and a subset $\rho$ of $X^{+} \times X^{+}$, and defines the semigroup $X^{+} / \rho^{\sharp}$. By standard convention, we 
may denote $(u, v) \in \rho$ by the equality $u=v$. Moreover, we may we identify the semigroup presentation with the semigroup that it defines.

Similarly, monoid presentations are given by the same notation $\langle X: \rho\rangle$ where $\rho \subseteq X^{*} \times X^{*}$ and define the monoid $X^{*} / \rho^{\sharp}$. In the case where $|u|=|v|$ for every $(u, v) \in \rho$, and hence for every $(w, x) \in \rho^{\sharp}$, we say that the corresponding presentation is homogeneous. In this article we will also need the notion of commutative semigroup and monoid presentations. To this end we denote by $\mathcal{C} X^{+}$ and $\mathcal{C} X^{*}$ the free commutative semigroup and free commutative monoid on $X$, respectively. We view $\mathcal{C} X^{+}$as consisting of all unordered non-empty words over $X$ under concatenation; similarly, $\mathcal{C} X^{*}$ consists of all unordered words over $X$.

Definition 2.5 A commutative semigroup presentation $\langle X: \rho\rangle$ consists of nonempty set $X$ and a subset $\rho$ of $\mathcal{C} X^{+} \times \mathcal{C} X^{+}$, and defines the commutative semigroup $\mathcal{C} X^{+} / \rho^{\sharp}$.

In Definition 2.5 we may think of $\rho$ as a subset of $X^{+} \times X^{+}$, if convenient. Commutative monoid presentations are defined in the obvious way.

\subsection{Monoid actions}

Throughout this subsection we let $M$ be a monoid. We briefly present the basic notions surrounding $M$-acts, in order to facilitate later discussions on coherency. Further details may be found in [18].

A set $A$ is a (right) $M$-act if there exists a map $A \times M \rightarrow A:(a, m) \mapsto a \cdot m$ such that for any $a \in A$ and $s, t \in M$ we have $a \cdot 1=a$ and $(a \cdot s) \cdot t=a \cdot(s t)$. An $M$ subact of an $M$-act $A$ is a subset $B$ of $A$ closed under the action of $M$; a congruence on $A$ is an equivalence relation $\theta$ on $A$ that is closed under the action of $M$, that is, for any $a, b \in A$ and $s \in M$, if $a \theta b$ then $a s \theta b s$. If $\theta$ is a congruence on $A$ then we may form the quotient $M$-act $A / \theta=\{[a]: a \in A\}$ with action $[a] s=[a s]$. Clearly, $M$ may be regarded as an $M$-act where the action is the multiplication in $M$ and the $M$ subacts are then precisely its right ideals. To avoid confusion, if $\theta$ is a congruence on the $M$-act $M$, then we refer to $\theta$ as a right congruence. Of course, if $M$ is commutative, then right congruences coincide with (monoid) congruences on $M$.

A congruence $\theta$ on an $M$-act $A$ is finitely generated if it is the smallest congruence containing a given finite set of elements of $A \times A$. An explicit formula for obtaining $\theta$ from its generators may be found in a way analogous to that in Equation (1). Note that the identity relation is always finitely generated. The notions of being free, finitely generated and finitely presented for an $M$-act $A$ are the standard ones from universal algebra, translated to the context of $M$-acts. Explicitly, (finitely generated) free $M$-acts are disjoint unions of (finitely many) copies of the $M$-act $M$; an $M$-act $A$ is finitely generated if $A=a_{1} M \cup \ldots \cup a_{n} M$ for some $a_{i} \in A, n \in \mathbb{N}^{0}$, and finitely presented if $A \cong F / \theta$ for some finitely generated free $M$-act $F$ and finitely generated congruence $\rho$ on $F$.

For any $M$-act, say $A$, and for every $a \in A$ we define the right congruence $r(a)$ on $M$ by 


$$
\boldsymbol{r}(a)=\{(u, v) \in M \times M: a u=a v\} .
$$

\subsection{Coherency and noetherianity}

A semigroup is weakly right Noetherian if every right ideal is finitely generated. This is a weaker condition than that of being right Noetherian, which means that every right congruence is finitely generated (a fact easily witnessed by considering a group). These are well-established notions, recently attracting new attention [22]. The following remark is worth stating explicitly.

Remark 2.6 Any weakly right noetherian semigroup is right ideal Howson.

In fact, we can weaken the hypothesis of Remark 2.6 much further.

Definition $2.7[9,26]$ A monoid $M$ is weakly right coherent if every finitely generated right ideal is finitely presented and right coherent if every finitely presented $M$-subact of every finitely presented $M$-act is finitely presented.

Any right noetherian monoid is right coherent, but weakly right noetherian monoids need not even be weakly right coherent [9]. We are interested in these notions here due to the result below, which may be regarded as analogous to that of Chase for rings [2].

Theorem 2.8 [9, Corollary 3.3, 3.4] A monoid $M$ is weakly right coherent if and only if for any $a, b \in M$ the right congruence $\boldsymbol{r}(a)$ is finitely generated, and the right ideal $a M \cap b M$ is finitely generated.

A monoid $M$ is right coherent if and only if for any finitely generated right congruence $\theta$ on $M$ and any $[a],[b] \in M / \theta$ we have the right congruence $\boldsymbol{r}([a])$ is finitely generated and the $M$-subact $[a] M \cap[b] M$ of $M / \theta$ is finitely generated.

Corollary 2.9 A weakly right coherent monoid is right ideal Howson.

\section{Examples of right ideal Howson semigroups}

We begin by summarising some previous remarks.

Remark 3.1 Any finite semigroup, (weakly) right noetherian semigroup or semigroup $S$ such that $S^{1}$ is (weakly) right coherent is right ideal Howson.

There are many examples of right coherent monoids [6, 9]. Rédei's Theorem states that the free commutative monoid $\mathcal{C} X^{*}$, for any finite set $X$, is (right) noetherian [23]. Gould used this result to show that for any set $X$, the free commutative monoid $\mathcal{C} X^{*}$ is (right) coherent [9, Theorem 4.3]. Our next result is a significant extension of the latter fact. The proof makes essential use of the fact that for a commutative monoid, right congruences and congruences coincide.

Theorem 3.2 Let $M$ be a monoid given by a commutative presentation $\langle X: \tau\rangle$, where $\tau$ is finite. Then $M$ is (right) coherent. 
Proof Let $\rho^{\sharp}$ be a finitely generated congruence on $M=\mathcal{C} X^{*} / \tau^{\sharp}$. We have that

$$
\rho=\left\{\left([a]_{\tau},[b]_{\tau}\right):(a, b) \in \sigma\right\}
$$

for some finite subset $\sigma$ of $\mathcal{C} X^{*} \times \mathcal{C} X^{*}$. We let $v=\tau \cup \sigma$ so that $v^{\sharp}$ is a finitely generated congruence on $\mathcal{C} X^{*}$. Taking care with the generators, it follows from the second isomorphism theorem [14, Theorem 1.5.4] that there exists a monoid isomorphism

$$
\theta: M / \rho^{\sharp} \rightarrow \mathcal{C} X^{*} / \nu^{\sharp}:\left[[w]_{\tau}\right]_{\rho} \theta=[w]_{v} .
$$

For convenience we denote the $\rho^{\sharp}$-class of $[w]_{\tau} \in M$ by $[w]_{\tau: \rho}$.

We are considering the monoid $M$ acting on the right of the $M$-act $M / \rho^{\sharp}$. To this end, note that for $[u]_{\tau} \in M$ and $[w]_{\tau: \rho} \in M / \rho^{\sharp}$ we have

$$
[w]_{\tau: \rho}[u]_{\tau}=\left[[w]_{\tau}\right]_{\rho}[u]_{\tau}=\left[[w]_{\tau}[u]_{\tau}\right]_{\rho}=\left[[w u]_{\tau}\right]_{\rho}=[w u]_{\tau: \rho} .
$$

Similarly, $\mathcal{C} X^{*}$ acts on $\mathcal{C} X^{*} / \nu^{\sharp}$ by $[w]_{v} u=[w u]_{v}$.

Proposition 3.3 Let $[w]_{\tau: \rho} \in M / \rho^{\sharp}$ and $[u]_{\tau},[v]_{\tau} \in M$, where $w, u, v \in \mathcal{C} X^{*}$. Then

$$
\left([u]_{\tau},[v]_{\tau}\right) \in \boldsymbol{r}\left([w]_{\tau: \rho}\right) \Longleftrightarrow(u, v) \in \boldsymbol{r}\left([w]_{v}\right) .
$$

Proof Let $w, u, v \in \mathcal{C} X^{*}$. From remarks above we have

$$
\begin{aligned}
\left([u]_{\tau},[v]_{\tau}\right) \in \boldsymbol{r}\left([w]_{\tau: \rho}\right) & \Longleftrightarrow[w u]_{\tau: \rho}=[w v]_{\tau: \rho} \\
& \Longleftrightarrow[w u]_{v}=[w v]_{v} \\
& \Longleftrightarrow(u, v) \in \boldsymbol{r}\left([w]_{v}\right)
\end{aligned}
$$

as required.

Let $w \in \mathcal{C} X^{*}$. Since $v$ is finite and $\mathcal{C} X^{*}$ is coherent, it follows that there exists a finite symmetric set of generators $\kappa$ for $\mathbf{r}\left([w]_{v}\right)$, say

$$
\kappa=\left\{\left(u_{i}, v_{i}\right): i \in \underline{n}\right\}
$$

for some $n \in \mathbb{N}^{0}$. We define

$$
\eta=\left\{\left(\left[u_{i}\right]_{\tau},\left[v_{i}\right]_{\tau}\right): i \in \underline{n}\right\},
$$

so that $\eta$ is also finite and symmetric.

Proposition 3.4 If $w \in \mathcal{C} X^{*}$ then, with the notation above, $\boldsymbol{r}\left([w]_{\tau: \rho}\right)=\langle\eta\rangle$.

Proof That $\eta \subseteq \boldsymbol{r}\left([w]_{\tau: \rho}\right)$ follows from Proposition 3.3.

Suppose for the converse that $\left([u]_{\tau},[v]_{\tau}\right) \in \boldsymbol{r}\left([w]_{\tau: \rho}\right)$, so that $(u, v) \in \boldsymbol{r}\left([w]_{v}\right)$, again by Proposition 3.3. Since $\kappa$ generates $\boldsymbol{r}\left([w]_{v}\right)$ there exists $\ell \in \mathbb{N}^{0}$ and a finite sequence of the form 


$$
u=z_{0}, z_{1}, \ldots, z_{\ell}=v
$$

where $z_{i-1}=c_{i} p_{i}$ and $z_{i}=c_{i} q_{i}$ with $\left(p_{i}, q_{i}\right) \in \kappa$ and $c_{i} \in \mathcal{C} X^{*}$ for all $i \in \underline{\ell}$. It follows that there is a sequence

$$
[u]_{\tau}=\left[z_{0}\right]_{\tau},\left[z_{1}\right]_{\tau}, \ldots,\left[z_{\ell}\right]_{\tau}=[v]_{\tau}
$$

where $\left[\left[z_{i-1}\right]_{\tau}=\left[c_{i} p_{i}\right]_{\tau}=\left[c_{i}\right]_{\tau}\left[p_{i}\right]_{\tau}\right.$ and $\left.\left[z_{i}\right]_{\tau}=\left[c_{i} q_{i}\right]_{\tau}=\left[c_{i}\right]_{\tau}\left[q_{i}\right]_{\tau}\right]$ with $\left(\left[p_{i}\right]_{\tau},\left[q_{i}\right]_{\tau}\right) \in$ $\eta$ for all $i \in \underline{\ell}$. Thus $\boldsymbol{r}\left([w]_{\tau: \rho}\right) \subseteq\langle\eta\rangle$ and we therefore have equality as desired.

Let $a, b \in \mathcal{C} X^{*}$ and let $I=[a]_{\tau: \rho} M \cap[b]_{\tau: \rho} M$ and $J=[a]_{\nu} \mathcal{C} X^{*} \cap[b]_{\nu} \mathcal{C} X^{*}$. We proceed to show that $I$ is finitely generated.

Proposition 3.5 If $w \in \mathcal{C} X^{*}$ then $[w]_{\tau: \rho} \in I \Longleftrightarrow[w]_{v} \in J$.

Proof Suppose that $w, u, v \in \mathcal{C} X^{*}$. Then

$$
\begin{aligned}
{[w]_{\tau: \rho}=[a]_{\tau: \rho}[u]_{\tau}=[b]_{\tau: \rho}[v]_{\tau} } & \Longleftrightarrow[w]_{\tau: \rho}=[a u]_{\tau: \rho}=[b v]_{\tau: \rho} \\
& \Longleftrightarrow \quad[w]_{v}=[a u]_{v}=[b v]_{v} \\
& \Longleftrightarrow \quad[w]_{v}=[a]_{v} u=[b]_{v} v
\end{aligned}
$$

as required.

Since $v$ is finitely generated, and $\mathcal{C} X^{*}$ is coherent, $J$ is generated by a set $\left\{[a c]_{v}\right.$ : $c \in Y\}$ for some finite subset $Y$ of $X^{*}$. Proposition 3.5 then gives us that $I$ is finitely generated by the set $\left\{[a c]_{\tau: \rho}: c \in Y\right\}$ and this completes the proof of Theorem 3.2.

From Corollary 2.9 and Theorem 3.2 we immediately deduce the following.

Corollary 3.6 Let $M$ be a monoid given by a commutative presentation $\langle X: \tau\rangle$, where $\tau$ is finite. Then $M$ is (right) ideal Howson.

In particular, any finitely presented commutative semigroup or monoid is (right) ideal Howson.

Changing tack, the following is well known.

Lemma 3.7 Let $S$ be an inverse semigroup. Then $S$ is right ideal Howson and satisfies $(R i)$ if and only if $i=1$.

Proof For any $a, b \in S$ we have $a S=a a^{-1} S$ and $b S=b b^{-1} S$. Since idempotents commute it follows that

$$
a S \cap b S=a a^{-1} S \cap b b^{-1} S=a a^{-1} b b^{-1} S .
$$

Since the free band on a finite set is finite (see [14]), any finitely generated band is finite. Hence:

Lemma 3.8 Every finitely generated band is right ideal Howson. 
Let $X$ be a countable set. For some $x, y \in X^{+}$, we say that a semigroup $S$ satisfies an identity $x=y$ if for every choice of homomorphism $\theta: X^{+} \rightarrow S$ we have $x \theta=y \theta$. A semigroup variety denotes a class of semigroups containing all semigroups that satisfy a given collection of identities.

Throughout we let $\mathcal{V}$ be a variety of bands. We recall the variety of right regular bands $\mathcal{R} \mathcal{R}$ (variety of rectangular bands $\mathcal{R B}$ ) is determined by the identities $x^{2}=x$ and $x y=y x y \quad\left(x^{2}=x\right.$ and $\left.x=x y x\right)$ (in addition to the identity guaranteeing associativity).

Theorem 3.9 Let $\mathcal{V}$ be a variety of bands. If $\mathcal{V} \subseteq \mathcal{R} \mathcal{R}$ or $\mathcal{V} \subseteq \mathcal{R} \mathcal{B}$, then every band $B \in \mathcal{V}$ is right ideal Howson. Further, $B$ satisfies (Ri) if and only if $i=1$, that is, $B$ satisfies Clifford's condition.

Proof Suppose that $B \in \mathcal{V}$. Since $B$ is a band, we note that for any $a, b \in B$ we have $a B \cap b B \subseteq a b B$. If $B \in \mathcal{R} \mathcal{R}$ then we have $a b \in a B$ and $a b=b a b \in b B$, so that in this case $a B \cap b B=a b B$ is principal. On the other hand, if $B \in \mathcal{R B}$ then for all $a, b \in B$ we have $a B \cap b B$ is either principal if $a \mathscr{R} b$ or empty else.

In order to prove the next therorem, we must draw upon Fennemore's result [8] concerning the defining identities of varieties of bands. This yields that if $\mathcal{V}$ is a variety of bands not contained in $\mathcal{R} \mathcal{R}$ or $\mathcal{R B}$, then $\mathcal{V}$ is defined by an identity $V:=p=q$ with the property that $\alpha(p)=\alpha(q)$ and $c(p)=c(q)$.

Theorem 3.10 Let $\mathcal{V}$ be a variety of bands not contained in $\mathcal{R} \mathcal{R}$ or $\mathcal{R} \mathcal{B}$. Then there exists a band $B_{\infty} \in \mathcal{V}$ that is not right ideal Howson, and for each $n \in \mathbb{N}^{0}$ a band $B_{n} \in \mathcal{V}$ that satisfies $(R n)$.

Proof Fix some $n \in \mathbb{N}^{0}$ and let $X_{n}=\left\{a, b, u_{i}, v_{i}: i \in \underline{n}\right\}$. If $n=0$ then we simply put $X_{0}=\{a, b\}$. Define two subsets $\rho_{n}$ and $\sigma_{n}$ of $X_{n}^{+} \times X_{n}^{+}$as follows

$$
\rho_{n}=\left\{\left(a u_{i}, b v_{i}\right): i \in \underline{n}\right\} \text { and } \sigma_{n}=\left\{\left(w, w^{2}\right): w \in X_{n}^{+}\right\} .
$$

In this way, if $n=0$ then $\rho_{0}=\emptyset$. Suppose $V$ is the defining identity for $\mathcal{V}$ (as above) and let $B_{n}$ be the band with semigroup presentation $\left\langle X_{n}: \rho_{n} \cup \sigma_{n} \cup V\right\rangle$. From this point we will write $X, \rho, \sigma$ and $B$ instead of $X_{n}, \rho_{n}, \sigma_{n}$ and $B_{n}$ respectively. Moreover, for ease of notation we will let $\tau=\rho \cup \sigma \cup V$.

It follows immediately from Lemma 3.8 that $B$ is right ideal Howson since it is finitely generated. Hence, it remains to show that $B$ satisfies $(R n)$. We achieve this by showing that $I=[a] B \cap[b] B$ is exactly $n$-generated.

Firstly, we suppose that there exist some $s, t \in X^{*}$ such that $(a s, b t) \in \tau^{\sharp}$. This implies that there exists a finite sequence of the form

$$
a s=z_{0}, z_{1}, \ldots, z_{\ell}=b t
$$

where $z_{i-1}=c_{i} p_{i} d_{i}$ and $z_{i}=c_{i} q_{i} d_{i}$ with $\left(p_{i}, q_{i}\right) \in \tau \cup \tau^{-1}$ and $c_{i}, d_{i} \in X^{*}$ for all $i \in \underline{\ell}$.

If $z_{i-1} \rightarrow z_{i}$ is an elementary $\sigma$ - or an elementary $V$-transition for all $i \in \underline{\ell}$, then we immediately reach a contradiction since $(w, x) \in(\sigma \cup V)^{\sharp}$ implies $\alpha(w)=\alpha(x)$. 
Therefore, we may assume that $z_{i-1} \rightarrow z_{i}$ is an elementary $\rho$-transition for some $i \in \underline{\ell}$, and, in order to avoid a similar contradiction, we may also assume that for at least one such $i \in \underline{\ell}$, we have $c_{i}=\epsilon$. Thus, we have shown $z_{i}=a u_{j} d_{i}$ or $b v_{j} d_{i}$ for some $i \in \underline{\ell}$ and $j \in \underline{n}$. It follows that

$$
I \subseteq \bigcup_{i \in \underline{n}}\left[a u_{i}\right] B
$$

the reverse inclusion is clear from the form of the presentation.

To show that $I$ is exactly $n$-generated, we note that if $w \tau^{\sharp} x$ for some $w, x \in X^{*}$ and $u_{i} \in c(w)$ then $\left\{u_{i}, v_{i}\right\} \cap c(x) \neq \emptyset$, since any elementary $\tau$-transition has this property. Thus if $i, j \in \underline{n}$ with $i \neq j$, then for no $t \in X^{*}$ do we have that $a u_{i} \tau^{\sharp} a u_{j} t$. Thus $\left[a u_{i}\right] B \nsubseteq\left[a u_{j}\right] B$ and so $I$ requires $n$ generators as claimed.

The existence of $B_{\infty}$ is proved in an entirely similar manner, starting with

$$
\rho_{\infty}=\left\{\left(a u_{i}, b v_{i}\right): i \in \mathbb{N}\right\}
$$

it is easy to see that $I=[a] B \cap[b] B$ cannot be finitely generated.

We end this section with a brief discussion of another finiteness condition for a monoid $M$, namely $\boldsymbol{R}$ [12]. This condition arises from axiomatisability properties of classes of right $M$-acts and states that for any $a, b \in M$ the subact of the direct product right $M$-act $M \times M$ given by

$$
\boldsymbol{R}(a, b)=\{(u, v) \in M \times M: a u=b v\}
$$

is finitely generated. It was shown that $\boldsymbol{R}$ is independent of being weakly right noetherian [12].

Lemma 3.11 If $M$ is a monoid satisfying $\boldsymbol{R}$ then $M$ is right ideal Howson.

Proof Let $a, b \in M$. It is clear that if $H$ is a finite set of generators for $\boldsymbol{R}(a, b)$, then $a K$ is a finite set of generators for $a M \cap b M$, where $K$ is the set of first co-ordinates of elements of $H$.

On the other hand, there certainly exist examples of semilattices that do not satisfy $\boldsymbol{R}$ [10]; since semilattices are inverse they are right and left ideal Howson.

The next corollary comes from Lemma 3.11 and the results of [10].

Corollary 3.12 The free inverse monoid, the free ample (restriction) monoid, and the free left ample (restriction) monoid on any set is right and left ideal Howson.

\section{Closure results}

In this section, we explore a number of closure results regarding the class of right ideal Howson semigroups. Our first result is immediate, since any free semigroup $X^{+}$is right ideal Howson.

Proposition 4.1 The class of right ideal Howson semigroups is not closed under morphic image. 


\subsection{Free products}

Let $\Lambda$ be a non-empty set and let $S_{\lambda}$ be a semigroup (monoid) for every $\lambda \in \Lambda$. Let

$$
X=\bigcup_{\lambda \in \Lambda} S_{\lambda}
$$

we write the product of $x, y \in X^{+}$or $X^{*}$ as $x \circ y$. Consider the subsets $\mu$ and $v$ of $X^{+} \times X^{+}$and $X^{*} \times X^{*}$, respectively, where

$$
\mu=\left\{(x \circ y, x y): x, y \in S_{\lambda} \text { for some } \lambda \in \Lambda\right\} \text { and } v=\mu \cup\left\{\left(1_{S_{\lambda}}, 1_{S_{\tau}}\right): \lambda, \tau \in \Lambda\right\},
$$

where $I_{S_{\lambda}}$ denotes the identity of $S_{\lambda}$ in the case of monoids. The free product of semigroups $S_{\lambda}, \lambda \in \Lambda$, may be given by the quotient $X^{+} / \mu^{\sharp}$, and the free product of monoids $S_{\lambda}, \lambda \in \Lambda$, may be given by the quotient $X^{*} / v^{\sharp}$. If $\Lambda=\underline{n}$ for some $n \in \mathbb{N}$, we may write $S_{1} \circ \cdots \circ S_{n}$ for the semigroup or monoid free product, where the distinction will be clear from the context.

Proposition 4.2 The class of right ideal Howson semigroups is closed under free products of semigroups.

Proof Let $S$ be the semigroup free product of right ideal Howson semigroups $S_{\lambda}, \lambda \in \Lambda$. Let $[a],[b] \in S$ where $[a]=\left[a_{1} \circ \cdots \circ a_{n}\right]$ and $[b]=\left[b_{1} \circ \cdots \circ b_{m}\right]$ and put $I=[a] S^{1} \cap[b] S^{1}$. We show that $I$ is finitely generated. We may assume that $n, m$ are least, so, for example, there does not exist an $i \in \underline{n-1}$ such that $a_{i} a_{i+1} \in S_{\lambda}$ for some $\lambda \in \Lambda$.

Either $I=\emptyset,[a] S^{1} \subseteq[b] S^{1},[b] S^{1} \subseteq[a] S^{1}$, or there exists some $[u],[v] \in S$ such that $[a \circ u]=[b \circ v]$. In the second and third cases $I$ is principal: we consider the final case. Here we take

$$
[u]=\left[u_{1} \circ \cdots \circ u_{k}\right] \text { and }[v]=\left[v_{1} \circ \cdots \circ v_{\ell}\right]
$$

for some least $k, \ell \in \mathbb{N}$, so that

$$
[a][u]=\left[a_{1} \circ \cdots \circ a_{n} \circ u_{1} \circ \cdots \circ u_{k}\right] \text { and }[b][v]=\left[b_{1} \circ \cdots \circ b_{m} \circ v_{1} \circ \cdots \circ v_{\ell}\right] .
$$

Let us assume $n \leq m$ and proceed with a case-by-case consideration.

(i) If $n<m$ then $a_{i}=b_{i}$ for all $i \in \underline{n-1}$ and $b_{n}=a_{n}$ or $b_{n}=a_{n} u_{1}$. In either case, $I=[b] S^{1}$ is principal. Dually if $m<n$.

(ii) If $n=m$ then again $a_{i}=b_{i}$ for all $i \in \underline{n-1}$. Suppose that $a_{n}, b_{n} \in S_{\lambda}$; if $a_{n}=b_{n}, a_{n}=b_{n} w$ or $b_{n}=a_{n} w$ for some $w \in S_{\lambda}^{1}$, then clearly $I$ is principal. If this is not the case, then $a_{n} u_{1}=b_{n} v_{1}$. Let $J=a_{n} S_{\lambda}^{1} \cap b_{n} S_{\lambda}^{1}$. Since $S_{\lambda}$ is right ideal Howson this intersection is finitely generated, say

$$
J=\bigcup_{i \in \underline{r}} a_{n} w_{i} S_{\lambda}^{1}
$$

Clearly 


$$
\bigcup_{i \in \underline{r}}\left[a_{1} \circ \ldots \circ a_{n} w_{i}\right] S_{\lambda}^{1} \subseteq I .
$$

On the other hand, $a_{n} u_{1}=a_{n} w_{i} w$ for some $i \in \underline{r}, w \in S_{\lambda}$, so that $[a] \circ[u] \in$ $\left[a_{1} \circ \ldots \circ a_{n} w_{i}\right] S^{1}$ for some $i \in \underline{r}$. Thus

$$
\bigcup_{i \in \underline{r}}\left[a_{1} \circ \ldots \circ a_{n} w_{i}\right] S^{1}=I
$$

so that $I$ is finitely generated as required.

The corresponding result hold for the free product of monoids.

Proposition 4.3 The class of right ideal Howson monoids is closed under free products.

Proof The argument runs along the same lines as that of Proposition 4.2, but with added technicalities due to the extra relations in $v$.

Let $S$ be the (monoid) free product of right ideal Howson monoids $S_{\lambda}, \lambda \in \Lambda$. Let $[a],[b] \in S$ and put $I=[a] S \cap[b] S$. We show that $I$ is finitely generated. If $[a]$ is the identity of $S$, or an element that has a right inverse, then the result is clear. Thus we may suppose $[a]=\left[a_{1} \circ \ldots \circ a_{n}\right]$ where $n \in \mathbb{N}, a_{i} \in S_{\lambda_{i}}, a_{i} \neq I_{S_{\lambda_{i}}}$ for $i \in \underline{n}$ and $\lambda_{i} \neq$ $\lambda_{i+1}$ for $i \in \underline{n-1}$. Now observe that if $n^{\prime}$ is greatest such that $a_{n^{\prime}}$ is not right invertible, then $[a] \mathscr{R}\left[a_{1} \circ \ldots \circ a_{n^{\prime}}\right]$. We may therefore assume from the outset that $a_{n} \in S_{\lambda_{n}}$ is not right invertible. Similarly, we can assume $[b]=\left[b_{1} \circ \ldots \circ b_{m}\right]$ where $m \in \mathbb{N}, b_{j} \in S_{\tau_{j}}, b_{j} \neq I_{S_{\tau_{j}}}$ for $j \in \underline{m}, \tau_{j} \neq \tau_{j+1}$ for $j \in \underline{m-1}$ and $b_{m} \in S_{\tau_{m}}$ is not right invertible.

If $I=\emptyset$, or if $[a]=[b][v]$ or $[b]=[a][u]$ for some $[u],[v] \in S$, then we are done. Suppose therefore that $[a][u]=[b][v]$ for some $[u],[v] \in S$. Given the fact that $a_{n}, b_{m}$ are chosen to be not right invertible, the proof proceeds as in that of Proposition 4.2.

\subsection{Direct and semidirect products}

We begin with a negative result for direct products of semigroups.

Proposition 4.4 The class of right ideal Howson semigroups is not closed under direct products.

Proof To see this, consider the free monogenic semigroup $S=\langle a\rangle$. Clearly $S$ is right ideal Howson since $S$ is (right) coherent, by Remark 3.1. Indeed, for any $n, m \in \mathbb{N}$ we have that $a^{n} S^{1} \cap a^{m} S^{1}=a^{k} S^{1}$ where $k=\max \{n, m\}$. Let $T=S \times S$. One may then easily verify that the intersection $I=(a, a) T^{1} \cap\left(a, a^{2}\right) T^{1}$ is generated by the set $K=\left\{\left(a^{2}, a^{k}\right): k \geq 3\right\}$ of $\mathscr{R}$-incomparable elements, and every generating set for $I$ must contain $K$.

On the other hand we have a positive result for right factorisable semigroups. 
Proposition 4.5 The class of right factorisable right ideal Howson semigroups is closed under direct products.

Proof Let $S$ and $T$ be right factorisable right ideal Howson semigroups. Notice that for any $s \in S$ and $t \in T$ we have $s S=s S^{1}, t T=t T^{1}$ and also

$$
(s, t)(S \times T)^{1}=(s, t)(S \times T)=s S \times t T=s S^{1} \times t T^{1} .
$$

It then follows easily that $S \times T$ is right ideal Howson.

If $S$ is free monogenic, then $S^{1} \times S^{1}$ is right ideal Howson from Proposition 4.5, but Proposition 4.4 tells us that $S \times S$ is not right ideal Howson. Hence:

Corollary 4.6 The class of right ideal Howson semigroups is not closed under taking subsemigroups.

We now turn our attention to semidirect products. Let $S$ and $T$ be semigroups such that $S$ acts on the left of $T$ via morphisms; we denote the resulting semidirect product by $T \rtimes S$. If the action of $S$ on $T$ is trivial, then $T \rtimes S$ is simply the direct product $T \times S$. Proposition 4.4 therefore tells us that the class of right ideal Howson semigroups is not closed under semidirect products. Considering now the case for monoids $S$ and $T$, where $S$ acts as a monoid on $T$ by monoid morphisms, the semidirect product $T \times S$ becomes a monoid. In view of Proposition 4.5 we know that the direct product of right ideal Howson monoids is right ideal Howson. By way of contrast we have the following.

Proposition 4.7 The class of right ideal Howson monoids is not closed under semidirect product.

Proof Let $X=\left\{a, b, a_{i}: i \in \mathbb{N}\right\}$ and $A=\left\{a_{i}: i \in \mathbb{N}\right\}$. We consider the left action of $X^{*}$ on $\mathcal{P}(X)$ where, for all $U \in \mathcal{P}(X)$, we have $b \cdot U=\emptyset=a_{i} \cdot U$ for all $i \in \mathbb{N}$ and

$$
a \cdot U=\left\{\begin{array}{cc}
\emptyset & U \cap A=\emptyset \\
\{a, b\} \cup U & \text { otherwise }
\end{array} .\right.
$$

One may verify that this is a left action of $X$ by monoid endomorphisms on the semilattice $\mathcal{P}(X)$ under union. The only case that needs thought is that of $a \cdot(U \cup$ $V$ ) where $U \cap A \neq \emptyset$ but $V \cap A=\emptyset$ (or the dual). In this case

$$
a \cdot(U \cup V)=\{a, b\} \cup U \cup V=\{a, b\} \cup U=a \cdot U \cup \emptyset=a \cdot U \cup a \cdot V,
$$

the second equality following since $V \subseteq\{a, b\}$. We may then extend the action of $X$ to that of the monoid $X^{*}$.

Let $S=\mathcal{P}(X) \rtimes X^{*}$ and let

$$
I=(\{a\}, a) S \cap(\{b\}, a) S .
$$

We claim that $I$ is not finitely generated.

To see this, notice that for any $i \in \mathbb{N}$ we have 


$$
Z_{i}:=\left(\left\{a, b, a_{i}\right\}, a\right)=(\{a\}, a)\left(\left\{a_{i}\right\}, \epsilon\right)=(\{b\}, a)\left(\left\{a_{i}\right\}, \epsilon\right) \in I .
$$

Further, if $(W, w) \in I$ then it is easy to see that $W=\{a, b\} \cup(W \cap A)$ where $W \cap A \neq \emptyset$, and $w=a w^{\prime}$ for some $w^{\prime} \in X^{*}$. Then if $a_{i} \in W$ we have

$$
(W, w)=\left(\left\{a, b, a_{i}\right\}, a\right)\left(W, w^{\prime}\right) .
$$

Since $Z_{i} \Varangle_{\mathscr{R}} Z_{j}$ for any $i \neq j$, it follows that $I$ cannot be finitely generated.

\section{Semigroup presentations}

In Corollary 3.6 we show that a commutative semigroup presentation $\langle X: \tau\rangle$, where $\tau$ is finite, gives rise to a (right) ideal Howson semigroup. In fact, one can show that both conditions - commutativity and the fact $\tau$ is finite - are strictly necessary. We illustrate this by way of the examples below.

Example 5.1 Let $S$ be given by the commutative semigroup presentation

$$
S=\left\langle a, b, u_{i}, v_{i}, i \in \mathbb{N}: a u_{i}=b v_{i}, i \in \mathbb{N}\right\rangle .
$$

Then $S$ is not ideal Howson.

Proof This presentation is the commutative semigroup version of the band presentation of $B_{\infty}$ given in Theorem 3.10. It is easy to see from the form of the presentation that

$$
[a] S^{1} \cap[b] S^{1}=\bigcup_{i \in \mathbb{N}}\left[a u_{i}\right] S^{1}
$$

and is not finitely generated.

It is also easy to see that the (non-commutative) semigroup on the same presentation as that in Example 5.1 is not right ideal Howson; the presentation, however, is not finite. We now give an example of a finitely presented semigroup that is not right ideal Howson.

Example 5.2 Let $S$ be given by the semigroup presentation

$$
S=\left\langle a, b, c, d, p, q, u, v: a u v c=b p q d, a u=u a, u b=b p, u v=u^{2} v^{2}\right\rangle .
$$

Then $S$ is not right ideal Howson.

Proof Let $X=\{a, b, c, d, p, q, u, v\}$ and define subsets $\rho$ and $\sigma$ of $X^{+} \times X^{+}$by

$$
\rho=\{(a u v c, b p q d)\} \text { and } \sigma=\left\{(a u, u a),(u b, b p),\left(u v, u^{2} v^{2}\right)\right\}
$$

and let $\tau=\rho \cup \sigma$. First, we note that for any $t \in X^{*}$ we have $\left[a u^{h} v c\right]=\left[a u^{k} v c t\right]$ if and only if $h=k$ and $t=\epsilon$. This is a fact witnessed by verifying, by induction on the length of a $\tau$-sequence, that 


$$
\left[a u^{h} v c\right]=\left\{u^{t} a u^{h+\ell-t} v^{\ell+1} c, u^{r} b p^{s} q d: \ell \geq 0,0 \leq t \leq h+\ell, r \geq 0, s \geq 0, r+s=h\right\} .
$$

It follows that $\left[a u^{h} v c\right]$ and $\left[a u^{k} v c\right]$, for any distinct $h, k \in \mathbb{N}$ are incomparable under the $\mathscr{R}$-order. Furthermore notice that $\left[a u^{h} v c\right] \in I=[a] S^{1} \cap[b] S^{1}$ for any $h \in \mathbb{N}$, since

$$
a u^{h} v c \sigma^{\sharp} u^{h-1} a u v c \rho^{\sharp} u^{h-1} b p q d \sigma^{\sharp} b p^{h} q d .
$$

To complete the proof, we must show that if $a w \tau^{\sharp} b x$ for some $w, x \in X^{*}$ then $[a w] \in\left[a u^{h} v c\right] S^{1}$ for some $h \geq 1$. Suppose therefore that $a w \tau^{\sharp} b x$, so there exists a finite sequence of the form

$$
a w=z_{0}, z_{1}, \ldots, z_{n}=b x
$$

where $z_{i-1}=c_{i} s_{i} d_{i}$ and $z_{i}=c_{i} t_{i} d_{i}$ with $c_{i}, d_{i} \in X^{*}$ and $\left(s_{i}, t_{i}\right) \in \tau \cup \tau^{-1}$ for all $i \in \underline{n}$. We claim that at least one elementary $\tau$-transition must be of the form

$$
z_{i}=u^{k} \text { auvcd }_{i}, z_{i+1}=u^{k} b p q d d_{i},
$$

where $k \geq 0, d_{i} \in X^{*}$. Suppose for contradiction that this is not the case; we show that for each $i \in \underline{n}^{0}$ we have $z_{i}=u^{k_{i}} a z_{i}^{\prime}$ for some $k_{i} \geq 0, z_{i}^{\prime} \in X^{*}$. Clearly this is true for $i=0$. Suppose for induction that $z_{i}=u^{k_{i}} a z_{i}^{\prime}$ as given where $i \in \underline{n}^{0}$. Avoiding the elementary $\tau$-transition of the form above, our possibilities for $z_{i} \rightarrow z_{i+1}$ are

$$
z_{i}=u^{k_{i}} a z_{i}^{\prime} \rightarrow u^{k_{i}-1} a u z_{i}^{\prime}=z_{i+1}, z_{i}=u^{k_{i}} a z_{i}^{\prime} \rightarrow u^{k_{i}+1} a z_{i}^{\prime \prime}=z_{i+1} \text { in case } z_{i}=u z_{i}^{\prime \prime}
$$

or

$$
z_{i}=u^{k_{i}} a z_{i}^{\prime} \rightarrow u^{k_{i}} a z_{i+1}^{\prime}=z_{i+1}
$$

where $z_{i}^{\prime} \rightarrow z_{i+1}^{\prime}$ under an elementary $\tau$-transition. Thus $\alpha\left(z_{n}\right) \in\{a, u\}$, a contradiction. Thus for some $i$ we must have $z_{i}=u^{k} a u v c d_{i}$ where $k \geq 0$, and then

$$
[a x]=\left[u^{k} a u v c d_{i}\right] \in\left[a u^{h} v c\right] S
$$

where $h=k+1 \in \mathbb{N}$. This completes the proof.

\subsection{Semigroup presentations of right and left ideal Howson semigroups}

We now turn to positive results, constructing semigroup presentations that are right (left, right and left) ideal Howson, which are, by construction, universal in a specific sense. The semigroups we construct in this subsection are also all cancellative.

For $n, m \in \mathbb{N}^{0}$ we define an alphabet $X_{n m}$ by

$$
X_{n m}=\left\{a, b, u_{i}, v_{i}, p_{j}, q_{j}: i \in \underline{n}, j \in \underline{m}\right\}
$$

and a relation $\rho_{n m}$ on $X_{n m}^{+}$by 


$$
\rho_{n m}=\left\{\left(a u_{i}, b v_{i}\right),\left(p_{j} a, q_{j} b\right): i \in \underline{n}, j \in \underline{m}\right\}
$$

and, as a convention, let $S_{\rho_{n n}}$ be the semigroup with presentation $\left\langle X_{n m}: \rho_{n m}\right\rangle$. If $n=0$ then we simplify our ingredients considerably; we have

$$
X_{0 m}=\left\{a, b, p_{j}, q_{j}: j \in \underline{m}\right\} \text { and } \rho_{0 m}=\left\{\left(p_{j} a, q_{j} b\right): j \in \underline{m}\right\}
$$

and similarly if $m=0$. If $m=n=0$ then $X=\{a, b\}$ and $\rho_{00}=\emptyset$. In this case, $S_{\rho_{00}}=\{a, b\}^{+}$, being free, is certainly right and left ideal Howson, with intersections of right (left) ideals being empty or principal.

Since $n, m \in \mathbb{N}^{0}$ are fixed, we simplify notation and denote $X_{n m}, \rho_{n m}$ and $S_{\rho_{n m}}$ by $X, \rho$ and $S$, respectively. To proceed we consider a specific factorisation of elements of $X^{+}$.

Let $U(n)=\left\{u_{i}, v_{i}: i \in \underline{n}\right\}$ and $P(m)=\left\{p_{j}, q_{j}: j \in \underline{m}\right\}$ where we regard $U(n)$ and $P(m)$ to be empty if $n=0$ and $m=0$ respectively. Let

$$
C(\rho)=\{p c, c u, p c u: c \in\{a, b\}, u \in U(n), p \in P(m)\}
$$

and notice that $C(\rho)$ is closed under $\rho^{\sharp}$. For any $w \in X^{+}$we may uniquely factorise $w$ as

$$
w=w_{0} r_{1} w_{1} \ldots w_{p-1} r_{p} w_{p}
$$

for $p \in \mathbb{N}^{0}$ and subject to the following conditions for all $i \in \underline{p}$ and $j \in \underline{p}^{0}$ :

(i) $r_{i} \in C(\rho)$;

(ii) $w_{j} \in X^{*}$ does not contain an element of $C(\rho)$ as a subword;

(iii) if $\alpha\left(r_{i}\right) \in\{a, b\}$ then $\omega\left(w_{i-1}\right) \notin P(m)$;

(iv) if $\omega\left(r_{i}\right) \in\{a, b\}$ then $\alpha\left(w_{i}\right) \notin U(n)$.

We call such a factorisation the $\rho$-factorisation of $w$ with corresponding $\rho$-length equal to $p$. Notice that $\left|r_{i}\right|=2$ or 3 for all $i \in \underline{p}$.

Claim 5.3 For $w, x \in X^{+}$have $w \rho^{\sharp} x$ if and only if $w, x$ have $\rho$-factorisations

$$
w=w_{0} r_{1} w_{1} \ldots w_{p-1} r_{p} w_{p} \text { and } x=w_{0} s_{1} w_{1} \ldots w_{p-1} s_{p} w_{p}
$$

where $r_{i} \rho^{\sharp} s_{i}$ for every $i \in \underline{p}$.

Proof If $w$ and $x$ are as given and $r_{i} \rho^{\sharp} s_{i}$ for every $i \in p$, then clearly $w \rho^{\sharp} x$.

For the converse, suppose that $w$ is as given and $w \rightarrow y$ is an elementary $\rho$ transition. then, (from the definition of $\rho$-factorisation) we must have $y=$ $w_{0} s_{1} w_{1} \ldots w_{p-1} s_{p} w_{p}$ where, for all but one $i \in \underline{p}$, we have $s_{i}=r_{i}$ and for a single $j \in \underline{p}$ we have $r_{j}, s_{j} \in C(\rho)$ with $r_{j} \rho^{\sharp} s_{j}$. Therefore $y$ has the form required. The result then follows by induction on the length of a $\rho$-sequence starting from $w$ and ending at $x$.

Claim 5.4 The semigroup $S$ is right and left ideal Howson. 
Proof We show that $S$ is right ideal Howson, the argument for left ideals being dual.

Let $w, x \in X^{+}$and put $I=[w] S^{1} \cap[x] S^{1}$. Suppose that $I \neq \emptyset$, so that $w h \rho^{\sharp} x k$ for some $h, k \in X^{*}$. By Claim 5.3 we have $\rho$-factorisations $w h=w_{0} r_{1} w_{1} \ldots w_{p-1} r_{p} w_{p}$ and $x k=w_{0} s_{1} w_{1} \ldots w_{p-1} s_{p} w_{p}$ for some $p \in \mathbb{N}^{0}$ where $r_{i} \rho^{\sharp} s_{i}$ for all $i \in p$. Without loss of generality we may assume $|w| \leq|x|$ and then consider all possible cases for the $\rho$-factorisation of $h$.

(i) Suppose that $w=w_{0} r_{1} w_{1} \ldots r_{i-1} s$ and $h=t r_{i} \ldots w_{p-1} r_{p} w_{p}$ where $w_{i}=s t$ for some $i \in \underline{p}^{0}$ and $s, t \in X^{*}$. Since $x=w_{0} s_{1} w_{1} \ldots s_{i-1}$ sy for some $y \in X^{*}$, we have that

$$
w y=w_{0} r_{1} w_{1} \ldots r_{i-1} s y \rho^{\sharp} w_{0} s_{1} w_{1} \ldots r s_{i-1} s y=x
$$

and so $I=[x] S^{1}$ is principal.

(ii) Suppose now that $w=w_{0} r_{1} w_{1} \ldots w_{i-1} s$ and $h=t w_{i} \ldots w_{p-1} r_{p} w_{p}$ where $r_{i}=$ st for some $i \in \underline{p}$ and $s, t \in X^{*}$. We write $x=w_{0} s_{1} w_{1} \ldots w_{i-1} s_{i} y$ for some $y \in X^{*}$ and see

$$
w t y=w_{0} r_{1} w_{1} \ldots w_{i-1} s t y=w_{0} r_{1} w_{1} \ldots w_{i-1} r_{i} y \rho^{\sharp} w_{0} s_{1} w_{1} \ldots w_{i-1} s_{i} y=x,
$$

so that $I=[x] S^{1}$ is principal.

(iii) Lastly, we suppose that $w$ is as in case (ii) and $x=w_{0} s_{1} w_{1} \ldots w_{i-1} u$ where $s_{i}=u v$ for some $i \in \underline{p}$ and $u, v \in X^{*}$ with $|s| \leq|u|$. If $s=u$ then $[w]=[x]$ and we are done. Otherwise, let us define

$$
R(\rho)=\left\{(p, q):(s p, u q) \in \rho^{\sharp} \cap(C(\rho) \times C(\rho))\right\} .
$$

It follows that $R(\rho)$ is finite since $C(\rho)$ is finite and certainly $w p \rho^{\sharp} x q$ for all $(p, q) \in R(\rho)$. Since $(t, v) \in R(\rho)$ we have

$$
I=\bigcup_{(p, q) \in R(\rho)}[w p] S^{1}
$$

and so $I$ is finitely generated.

Claim 5.5 The semigroup S satisfies $(\mathrm{Rn})$ and $(\mathrm{Lm})$. Moreover, the intersection of any two principal right (left) ideals of $S$ requires at most $n(m)$ generators.

Proof Again we only give the proof for right ideals. Let $w, x \in X^{+}$; we show the intersection $I=[w] S^{1} \cap[x] S^{1}$ requires at most $n$ generators. The only situation where $I$ is not empty or principal is in the second situation of (iii). Here we consider all the possibilities for $(p, q) \in R(\rho)$; we can have $\left|r_{i}\right|=\left|s_{i}\right|=2$ with $|s|=|u|=1$ or $|s|=1$ and $|u|=2$, or $\left|r_{i}\right|=\left|s_{i}\right|=3$ with $|s|=|u|=1$ or $|s|=1$ and $|u|=2$, or 3 , or $|s|=2$ and $|u|=2$ or 3 . A case-by-case analysis for all the possibilities, paying special attention to the case where $m=0$ or $n=0$ or both, now gives the result. In particular, when $n \geq 1$ with $w=a$ and $x=b$ we have 


$$
I=\bigcup_{i \in \underline{n}}\left[a u_{i}\right] S^{1}
$$

which achieves the bound $n$ as required.

We are now in a position to given the main result of this subsection.

Theorem 5.6 Let $n, m \in \mathbb{N}^{0}$. The semigroup $S_{\rho_{n m}}$ is cancellative, right and left ideal Howson, and satisfies $(\mathrm{Rn})$ and $(\mathrm{Lm})$. Further, the intersection of any two principal right (left) ideals of $S$ requires at most $n(m)$ generators.

Proof Cancellativity follows from the conditions given by Adjan in [1] for a semigroup given by a presentation to be embeddable into a group. The remainder of the result comes from Claims 5.3, 5.4 and 5.5.

We now show that our semigroup $S_{\rho_{n m}}$ is in a specific sense universal.

Proposition 5.7 Let $n, m \in \mathbb{N}^{0}$. Suppose $U$ is a semigroup containing elements $\alpha, \beta$ such that $\alpha U^{1} \cap \beta U^{1}$ and $U^{1} \alpha \cap U^{1} \beta$ are each exactly $n$ - and m-generated, respectively, by $\alpha \gamma_{1}=\beta \delta_{1}, \ldots, \alpha \gamma_{n}=\beta \delta_{n}$ and $\mu_{1} \alpha=\pi_{1} \beta, \ldots, \mu_{m} \alpha=\pi_{m} \beta$, respectively. Then there is a homomorphism $\theta: S_{\rho_{n m}} \rightarrow U$ such that

$$
[a] \theta=\alpha,[b] \theta=\beta,\left[u_{i}\right] \theta=\gamma_{i},\left[v_{i}\right] \theta=\delta_{i},\left[p_{j}\right] \theta=\mu_{j} \text { and }\left[q_{j}\right] \theta=\pi_{j}
$$

for all $i \in \underline{n}$ and $j \in \underline{m}$.

Proof Let $\psi: X_{n m}^{+} \rightarrow U$ be given by determining its values on the elements of $X_{n m}$ by

$$
a \psi=\alpha, b \psi=\beta, u_{i} \psi=\gamma_{i}, v_{i} \psi=\delta_{i}, p_{j} \psi=\mu_{j} \text { and } q_{j} \psi=\pi_{j}
$$

for all $i \in \underline{m}$ and $j \in \underline{n}$. Clearly $\rho_{n m} \subseteq \operatorname{ker} \psi$ so that $\psi$ induces a morphism $\theta$ : $S_{\rho_{n m}} \rightarrow U$ as in the statement of the proposition.

Remark 5.8 Let $n \geq 1$. The semigroup $S_{\rho_{1 m}}$ is not finitely right aligned. To see this, suppose that

$$
[a] S \cap[b] S=\left[w_{1}\right] S \cup \ldots \cup\left[w_{\ell}\right] S
$$

for some $w_{k} \in X^{+}$such that $\left[w_{k}\right] \in[a] S^{1} \cap[b] S^{1}$ for all $k \in \underline{\ell}$. We have $\left[a u_{1}\right]=\left[b v_{1}\right] \in[a] S \cap[b] S$, so that $\left[a u_{1}\right]=\left[b v_{1}\right]=\left[w_{k}\right][z]$ for some $k \in \underline{\ell}$ and $z \in X^{+}$. We must have that $w_{k} z=a u_{i}$ or $b v_{i}$. If $w_{k}=a$ then we would not have $w_{k} \in[b] S^{1}$ and similarly if $w_{k}=b$, a contradiction.

In fact, an easier approach to obtain a semigroup presentation that satisfies $(R n)$ and $(L m)$ for some fixed $n, m \in \mathbb{N}^{0}$, but producing a less tight result, runs as follows.

For $n, m \in \mathbb{N}^{0}$ we define an alphabet $Y_{n m}$ by

$$
Y_{n m}=\left\{a, b, c, d, u_{i}, v_{i}, p_{j}, q_{j}: i \in \underline{n}, j \in \underline{m}\right\}
$$

and a relation $\sigma_{m n}$ on $Y_{m n}^{+}$by 


$$
\sigma_{n m}=\left\{\left(a u_{i}, b v_{i}\right),\left(p_{j} c, q_{j} d\right): i \in \underline{n}, j \in \underline{n}\right\}
$$

and, as a convention, we let $T_{\sigma_{n m}}$ be the semigroup with presentation $\left\langle Y_{n m}: \sigma_{n m}\right\rangle$. If $n=0$ then (as before) we have

$$
Y_{0 m}=\left\{a, b, c, d, p_{j}, q_{j}: j \in \underline{m}\right\} \text { and } \sigma_{0 m}=\left\{\left(p_{j} c, q_{j} d\right): j \in \underline{m}\right\}
$$

and similarly when $m=0$. If in fact $n=m=0$ then we simply have $Y_{00}=$ $\{a, b, c, d\}$ and $\sigma_{00}=\emptyset$. The proof for the following theorem is similar to that of Theorem 5.6 but rather simpler, since the complications of overlapping generators for $\sigma_{n m}^{\sharp}$ do not occur.

Theorem 5.9 Let $n, m \in \mathbb{N}^{0}$. The semigroup $T_{\sigma_{n m}}$ is cancellative, right and left ideal Howson, and satisfies $(\mathrm{Rn})$ and $(\mathrm{Lm})$. Further, the intersection of any two principal right (left) ideals of $S$ requires at most $n(m)$ generators.

There is also a corresponding universal type result for $T_{\sigma_{n m}}$, analogous to that of Proposition 5.7. Namely, if $U$ is a semigroup containing elements $\alpha, \beta, \gamma$ and $\delta$ such that $\alpha U^{1} \cap \beta U^{1}$ and $U^{1} \gamma \cap U^{1} \delta$ are each exactly $n$ - and $m$-generated respectively, then $U^{1}$ contains a morphic image of $T_{\sigma_{n m}}$ obtained as in Proposition 5.7.

\subsection{Commutative semigroup presentations of (right) ideal Howson semigroups}

For any $n, m \in \mathbb{N}^{0}$, the semigroup $S_{\rho_{n m}}$ is not commutative. In this section, we provide a commutative semigroup presentation that satisfies $(R n)$, which now coincides with $(L n)$, for any fixed $n \in \mathbb{N}$. Unlike the case for $S_{\rho_{n m}}$ we do not automatically have that the semigroups we construct are cancellative. However, we can construct natural quotients that satisfy $(R n)$ and are cancellative.

For a fixed $n \in \mathbb{N}^{0}$ we define the alphabet $X_{n}$ by

$$
X_{n}=\left\{a, b, u_{i}, v_{i}: i \in \underline{n}\right\}
$$

and the relations $\tau_{n}$ and $v_{n}$ on $X_{n}^{+}$by

$$
\tau_{n}=\left\{\left(a u_{i}, b v_{i}\right): i \in \underline{n}\right\}, v_{n}=\left\{\left(a u_{i}, b v_{i}\right),\left(u_{i} v_{j}, u_{j} v_{i}\right): i, j \in \underline{n}, i \neq j\right\} .
$$

Similarly to the conventions in Sect. 5.1, we let $S_{\tau_{n}}$ and $S_{v_{n}}$ be the semigroups with commutative presentations $\left\langle X_{n}: \tau_{n}\right\rangle$ and $\left\langle X_{n}: v_{n}\right\rangle$ respectively. If $n=0$ then we simply put $X_{0}=\{a, b\}$ and

$$
\tau_{0}=\{(a b, b a),(b a, a b)\}=v_{0} .
$$

Since $n \in \mathbb{N}^{0}$ is fixed, we will write $X, \tau, v, S_{\tau}$ and $S_{v}$ instead of $X_{n}, \tau_{n}, v_{n}, S_{\tau_{n}}$ and $S_{v_{n}}$ respectively. Also, note that we will continue to refer to words but we now mean elements of $\mathcal{C} X^{+}$rather than $X^{+}$.

Theorem 5.10 The commutative semigroups $S_{\tau_{n}}$ and $S_{v_{n}}$ satisfy $(R(n+1))$. 
Proof From Corollary 3.6, we have that both $S_{\tau}$ and $S_{v}$ are right and left ideal Howson. A straightforward argument, using the fact that both presentations are homogeneous, verifies that

$$
[a]_{\kappa} S_{\kappa}^{1} \cap[b]_{\kappa} S_{\kappa}^{1}=[a b]_{\kappa} S_{\kappa}^{1} \cup \bigcup_{i \in \underline{n}}\left[a u_{i}\right]_{\kappa} S^{1}
$$

for $\kappa=\tau$ or $\kappa=v$. Further, in each case, no given generator is redundant.

Notice that in $S_{\tau}$ for any $i, j \in \underline{n}$ we have $\left[a u_{i} v_{j}\right]_{\tau}=\left[a u_{j} v_{i}\right]_{\tau}$ but if $i \neq j$ then $\left[u_{i} v_{j}\right]_{\tau} \neq\left[u_{j} v_{i}\right]_{\tau}$, so that if $n \geq 2$ the semigroup $S_{\tau}$ is not cancellative. However, we now set out to show that $S_{v}$ is cancellative.

We begin by making some immediate observations about words in the same $\tau^{\sharp}$ class or $v^{\sharp}$-class.

Remark 5.11 For $w, x \in \mathcal{C} X^{+}$with $w \tau^{\sharp} x$ or $w v^{\sharp} x$ we notice that

(i) $|w|=|x|$ since the presentations are homogeneous;

(ii) we may write $w=a^{p_{0}} b^{q_{0}} u_{1}^{p_{1}} v_{1}^{q_{1}} \ldots u_{n}^{p_{n}} v_{n}^{q_{n}}$ for some $p_{i}, q_{i} \in \mathbb{N}^{0}$ for all $i \in \underline{n}^{0}$.

With this in mind, for $w \in X^{+}$we define $K(w)$ and $k_{i}(w)$ to be

$$
K(w)=|w|_{b}+\sum_{i \in \underline{n}}|w|_{u_{i}}, k_{0}(w)=|w|_{a}+|w|_{b} \text { and } k_{i}(w)=|w|_{u_{i}}+|w|_{v_{i}}
$$

for all $i \in \underline{n}$. Notice that for any $w, x \in \mathcal{C} X^{+}$we have

$$
K(w x)=K(w)+K(x) \text { and } k_{i}(w x)=k_{i}(w)+k_{i}(x)
$$

for any $i \in \underline{n}^{0}$. We say that $w, x \in \mathcal{C} X^{+}$are balanced if $k_{i}(w)=k_{i}(x)$ for all $i \in \underline{n}^{0}$. The next claim is clear from the definition of $\tau$ and $v$.

Claim 5.12 If $w, x \in X^{+}$are such that $w \tau^{\sharp} x$ or $w v^{\sharp} x$, then $w$ and $x$ are balanced and $K(w)=K(x)$.

Any easy argument gives:

Claim 5.13 If $w, x \in X^{+}$are such that $k_{0}(w), k_{0}(x)>0$, then $w \tau^{\sharp} x$ if and only if $w v^{\sharp} x$.

Since $\tau$ and $v$ are homogeneous relations on $\mathcal{C} X^{+}$, the question follows of when $w \tau^{\sharp} x$ or $w v^{\sharp} x$ is decidable for $w, x \in \mathcal{C} X^{+}$. To enable our proof of cancellativity, we establish explicitly the existence of a normal form in each $\tau^{\sharp}$-class and $v^{\sharp}$-class. First, we introduce a linear order on words in $\mathcal{C} X^{+}$. Let $w, x \in \mathcal{C} X^{+}$where

$$
w=a^{p_{0}} b^{q_{0}} u_{1}^{p_{1}} v_{1}^{q_{1}} \ldots u_{n}^{p_{n}} v_{n}^{q_{n}} \text { and } x=a^{r_{0}} b^{s_{0}} u_{1}^{r_{1}} v_{1}^{s_{1}} \ldots u_{n}^{r_{n}} v_{n}^{s_{n}}
$$

We say that $w \leq x$ if and only if there exists some $i \in \underline{n}^{0}$ such that $p_{j}=r_{j}$ for all $j<i$ and $p_{i}<r_{i}$ (or in fact $p_{0}<r_{0}$ ). One may verify the following.

Claim 5.14 The relation $\leq$ is a partial order on $\mathcal{C X}^{+}$. 
In particular, $\leq$ is a partial order when restricted to any set of balanced words; certainly any $\tau^{\sharp}$-class or $v^{\sharp}$-class. Hence, let $w^{\tau}$ and $w^{v}$ be the unique words in $[w]_{\tau}$ and $[w]_{v}$ that are greatest under $\leq$. We now draw upon some well-known results regarding rewriting systems to obtain $w^{\tau}$ and $w^{v}$. For basic definitions surrounding rewriting systems we refer the reader to the work of Gray and Malheiro [13].

Claim 5.15 The rewriting system on $[w]_{\tau}$ for all $w \in X^{+}$, given by the rewriting rules $b v_{k} \rightarrow a u_{k}$ and $a v_{i} u_{j} \rightarrow a u_{i} v_{j}$ for all $i, j, k \in \underline{n}$ with $i<j$, is confluent.

Proof If $w=y b v_{k}$ and $x=y a u_{k}$ for some $y \in X^{*}$, then clearly $w \tau^{\sharp} x$ and $w<x$. Correspondingly, if $w=y a v_{i} u_{j}$ and $x=y a u_{i} v_{j}$ for some $y \in X^{*}$ with $i<j$ then again $w \tau^{\sharp} x$ and $w<x$. It follows that this is a noetherian rewriting system. It is routine to check that it is also locally confluent and thus confluent.

Consequently, if $w \in \mathcal{C} X^{+}$, then applying the rewriting rules to $w$ yields a unique reduced word $x \in[w]_{\tau}$, and $x$ is independent of the choice of $w$. We know that $x \leq w^{\tau}$ and we deduce from $w^{\tau} \leq x^{\tau}=x$ that $x=w^{\tau}$. We say that $w^{\tau}$ is the word in normal form in $[w]_{\tau}$. An entirely similar argument can be made for a rewriting system consisting of rewriting rules $b v_{k} \rightarrow a u_{k}$ and $v_{i} u_{j} \rightarrow u_{i} v_{j}$ (for all $i, j, k \in \mathbb{N}$ such that $i \leq j$ ) on elements of $[w]_{v}$ for any $w \in \mathcal{C} X^{+}$.

Claim 5.16 If $w^{\tau}$ is in normal form in the $\tau^{\sharp}$-class of $w$, then it must be one of the following types:

(F1a) $w^{\tau}=a^{p_{0}} u_{1}^{p_{1}} u_{2}^{p_{2}} \ldots u_{i-1}^{p_{i-1}} u_{i}^{p_{i}} v_{i}^{q_{i}} v_{i+1}^{q_{i+1}} \ldots v_{n}^{q_{n}}$ for $p_{0}>0$ and $q_{i}>0$ or $i-1=n$;

(F2) $\quad w^{\tau}=a^{p_{0}} b^{q_{0}} u_{1}^{p_{1}} u_{2}^{p_{2}} \ldots u_{n}^{p_{n}}$ for $q_{0}>0$;

$$
w^{\tau}=u_{1}^{p_{1}} v_{1}^{q_{1}} \ldots u_{n}^{p_{n}} v_{n}^{q_{n}} .
$$

Similarly, if $w^{v}$ is in normal form in the $v^{\sharp}$-class of $w$, then either it is type $(\mathrm{F} 1 b)$ or (F2) where

(F1b) $w^{v}=a^{p_{0}} u_{1}^{p_{1}} u_{2}^{p_{2}} \ldots u_{i-1}^{p_{i-1}} u_{i}^{p_{i}} v_{i}^{q_{i}} v_{i+1}^{q_{i+1}} \ldots v_{n}^{q_{n}}$ for $q_{i}>0$ or $i-1=n$.

To see this, one can verify that it is not possible to apply a rewriting rule (from their respective rewriting systems) to $w^{\tau}$ or $w^{v}$ as above. We now give a partial converse to Claim 5.12 .

Claim 5.17 Suppose $w, x \in X^{+}$are in normal form and balanced with $k_{0}(w)=k_{0}(x)>0$.

(i) If $|w|_{a}<|x|_{a}$ then $|w|_{u_{\ell}} \geq|x|_{u_{\ell}}$ for all $\ell \in \underline{n}$ and $K(w)-K(x)>0$;

(ii) if $|w|_{a}=|x|_{a}$ and $|w|_{u_{k}}>|x|_{u_{k}}$ for some $k \in \underline{n}$, then $|w|_{u_{\ell}} \geq|x|_{u_{\ell}}$ for all $\ell \in \underline{n}$ and $K(w)-K(x)>0$.

Proof Suppose that $w, x \in X^{+}$as in the statement above.

(i) If $|w|_{a}<|x|_{a}$, then as $k_{0}(x)>0$, we must have that 


$$
w=a^{p_{0}} b^{q_{0}} u_{1}^{p_{1}} u_{2}^{p_{2}} \ldots u_{n}^{p_{n}} \text { for } q_{0}>0
$$

is of type (F2). Either

$$
x=a^{r_{0}} b^{s_{0}} u_{1}^{p_{1}} u_{2}^{p_{2}} \ldots u_{n}^{p_{n}} \text { for } s_{0}>0
$$

is also of type $(\mathrm{F} 2)$ or

$$
x=a^{r_{0}} u_{1}^{p_{1}} u_{2}^{p_{2}} \ldots u_{i-1}^{p_{i-1}} u_{i}^{r_{i}} v_{i}^{s_{i}} v_{i+1}^{p_{i+1}} \ldots v_{n}^{p_{n}} \text { for } r_{0}>0, s_{i}>0 \text { or } i-1=n
$$

is of type (F1a). In both cases we see that certainly $|w|_{u_{\ell}} \geq|x|_{u_{\ell}}$ for all $\ell \in \underline{n}$. Since $|w|_{a}<|x|_{a}$ if and only if $|w|_{b}>|x|_{b}$ it is then clear that $K(w)-K(x)>0$.

(ii) Suppose that $|w|_{a}=|x|_{a}$ and $|w|_{u_{k}}>|x|_{u_{k}}$ for some $k \in \underline{n}$. Since $|w|_{a}=|x|_{a}$, either $w$ and $x$ are both of type (F1a) or both of type (F2). In the latter case, it is easy to see that $w=x$, contradicting the hypothesis. Thus

$$
w=a^{p_{0}} u_{1}^{p_{1}} u_{2}^{p_{2}} \ldots u_{i-1}^{p_{i-1}} u_{i}^{p_{i}} v_{i}^{q_{i}} v_{i+1}^{q_{i+1}} \ldots v_{n}^{q_{n}} \text { for } p_{0}>0, q_{i}>0 \text { or } i-1=n
$$

and

$$
x=a^{p_{0}} u_{1}^{r_{1}} u_{2}^{r_{2}} \ldots u_{j-1}^{r_{j-1}} u_{j}^{r_{j}} v_{j}^{s_{j}} v_{j+1}^{s_{j+1}} \ldots v_{n}^{s_{n}} \text { for } p_{0}>0, s_{j}>0 \text { or } j-1=n
$$

are of type (F1a). We know that $|w|_{u_{k}}>|x|_{u_{k}}$ for some $k \in \underline{n}$, and the only way this can occur is if $i>j$ or $i=j$ and $p_{i}>r_{i}$. In either case, $|w|_{u_{\ell}} \geq|x|_{u_{\ell}}$ for all $\ell \in \underline{n}$. Again, it is clear that $K(w)-K(x)>0$.

The next claim is now immediate.

Claim 5.18 Suppose $w, x \in X^{+}$are in normal form and balanced with $k_{0}(w)=k_{0}(x)>0$. Then $w=x$ (equivalently $w \tau^{\sharp} x$ ) if and only if $K(w)=K(x)$.

Theorem 5.19 The semigroup $S_{v}$ is cancellative.

Proof To see that $S_{v}$ is cancellative, suppose that $[w]_{v},[x]_{v},[h]_{v} \in S_{v}$ with $[w]_{v}[h]_{v}=[x]_{v}[h]_{v}$. We may assume that $w, x, h$ are in normal form.

We have $w h v^{\sharp} x h$ so that

$$
k_{i}(w)+k_{i}(h)=k_{i}(w h)=k_{i}(x h)=k_{i}(x)+k_{i}(h),
$$

giving $k_{i}(w)=k_{i}(x)$ for all $i \in \underline{n}^{0}$ and, similarly, $K(w)=K(x)$.

If $k_{0}(w)=k_{0}(x)>0$, then by Claim 5.13 we have $w \tau^{\sharp} x$ if and only if $w v^{\sharp} x$, so that $w=x$ from Claim 5.18.

Suppose therefore that $k_{0}(w)=k_{0}(x)=0$; it follows that $w, x$ have form $(\mathrm{F} 1 b)$. Using the fact that $w, x$ are balanced and $K(w)=K(x)$ a now familiar analysis again gives us that $w=x$. Certainly in each case we have $[w]_{v}=[x]_{v}$.

Our semigroups $S_{\tau}$ and $S_{v}$ have universal properties corresponding to those for $S_{\tau}$ in Proposition 5.7. 
Proposition 5.20 Let $S$ be a commutative (commutative and cancellative) semigroup such that $S$ contains two principal right ideals $\alpha S^{1}$ and $\beta S^{1}$ such that $\alpha S^{1} \cap \beta S^{1}$ has exactly $n$ generators $\alpha \gamma_{1}=\beta \delta_{1}, \ldots, \alpha \gamma_{n}=\beta \delta_{n}$. Then there is a homomorphism $\theta: S_{\tau} \rightarrow S\left(\theta: S_{v} \rightarrow S\right)$ such that $[a] \theta=\alpha,[b] \theta=\beta,\left[u_{i}\right] \theta=\gamma_{i}$ and $\left[v_{i}\right] \theta=\delta_{i}$ for all $i \in \underline{n}$.

Open Access This article is licensed under a Creative Commons Attribution 4.0 International License, which permits use, sharing, adaptation, distribution and reproduction in any medium or format, as long as you give appropriate credit to the original author(s) and the source, provide a link to the Creative Commons licence, and indicate if changes were made. The images or other third party material in this article are included in the article's Creative Commons licence, unless indicated otherwise in a credit line to the material. If material is not included in the article's Creative Commons licence and your intended use is not permitted by statutory regulation or exceeds the permitted use, you will need to obtain permission directly from the copyright holder. To view a copy of this licence, visit http:// creativecommons.org/licenses/by/4.0/.

\section{References}

1. Adjan, S.I.: Defining relations and algorithmic problems for groups and semigroups. Trudy Mat. Inst. Akad. Nauk SSSR. 85 (1966)

2. Chase, S.U.: Direct product of modules. Trans. Am. Math. Soc. 97, 457-473 (1960)

3. Cherubini, A., Petrich, M.: The inverse hull of right cancellative semigroups. J. Algebra 111, 74-113 (1987)

4. Clifford, A.H.: A class of $d$-simple semigroups. Am. J. Math. 75, 547-556 (1953)

5. Clifford, A.H., Preston, G.B.: The algebraic theory of semigroups, Volume 1. Am. Math. Soc. (1967)

6. Dandan, Y., Gould, V., Hartmann, M., Ruškuc, N., Zenab, R.: Coherence and constructions for monoids. arXiv:1906.05515

7. Exel, R., Steinberg, B.: Representations of the inverse hull of a 0-left cancellative semigroup. arXiv: 1802.06281

8. Fennemore, C.: All varieties of bands. Semigroup Forum 1, 172-179 (1970)

9. Gould, V.: Coherent monoids. J. Aust. Math. Soc. 53, 166-182 (1992)

10. Gould, V., Hartmann, M.: Coherency, free inverse monoids and related free algebras. Math. Proc. Camb. Philos. Soc. 163, 23-45 (2017)

11. Gould, V., Hartmann, M., Ruškuc, N.: Free monoids are coherent. Proc. Edinb. Math. Soc. 60, 127-131 (2017)

12. Gould, V., Hartmann, M., Shaheen, L.: On some finitary questions arising from the axiomatisability of certain classes of monoid acts. Commun. Algebra 42, 2584-2602 (2014)

13. Gray, R., Malheiro, A.: Finite complete rewriting systems for regular semigroups. Theor. Comput. Sci. 412, 654-661 (2011)

14. Howie, J.M.: Fundamentals of Semigroup Theory. Oxford University Press, Oxford (1995)

15. Howson, A.G.: On the intersection of finitely generated free groups. J. Lond. Math. Soc. 29, 428-434 (1954)

16. Jones, P.R.: A note on the Howson property in inverse semigroups. Bull. Aust. Math. Soc. 94, 457-463 (2016)

17. Jones, P.R., Trotter, P.G.: The Howson property for free inverse semigroups. Simon Stevin 63, 277-284 (1989)

18. Kilp, M., Knauer, U., Mikhalev, A.V.: Monoids, Acts, and Categories. De Gruyter Exp. Math. 29 (2000)

19. Lawson, M.L.: Contructing inverse semigroups from category actions. J. Pure Appl. Algebra 137, 57-101 (1999)

20. Lawson, M.V., Vdovina, A.: The universal Boolean inverse semigroup presented by the abstract Cuntz-Krieger relations. arXiv:1902.02583

21. McAlister, D.B.: One-to-one partial right translations of a right cancellative semigroup. J. Algebra 43, 231-251 (1976) 
22. Miller, C., Ruškuc, N.: Right noetherian semigroups. Int. J. Algebra Comput. 30, 13-48 (2019)

23. Rédei, L.: Theorie der endlich erzeugbaren kommutativen Halbgruppen. Physica-Verlag, Berlin (1963)

24. Reilly, N.R.: Bisimple inverse semigroups. Trans. Am. Math. Soc. 132, 101-114 (1968)

25. Silva, P.V., Soares, F.: Howson's property for semidirect products of semilattices by groups. Commun. Algebra 44, 2482-2494 (2016)

26. Wheeler, W.H.: Model companions and definability in existentially complete structures. Isr. J. Math. 25, 305-330 (1976)

Publisher's Note Springer Nature remains neutral with regard to jurisdictional claims in published maps and institutional affiliations. 